\title{
Métodos de neuroimagem e abuso de substâncias psicoativas
}

\author{
Sergio Nicastri
}

Grupo Interdisciplinar de Estudos de Álcool e Drogas (GREA) do Departamento de Psiquiatria da Faculdade de Medicina da Universidade de São Paulo (FMUSP)

\section{Introdução}

A Organização Mundial da Saúde (OMS) considera o consumo de substâncias psicoativas um problema crescente de saúde pública, tanto em países desenvolvidos quanto nos em desenvolvimento. ${ }^{1}$ No Brasil, o alcoolismo é o transtorno mental mais freqüente entre a população masculina. ${ }^{2}$ Diante desse quadro, fica mais clara a importância de se conhecer melhor a interação entre as diferentes substâncias de abuso e o sistema nervoso central (SNC), tanto em termos de efeitos agudos, quanto em termos de conseqüências a longo prazo.

Os métodos de imagem empregados nos artigos discutidos incluem técnicas tomográficas por emissão, como a tomografia por emissão de pósitrons (PET) e a tomografia computadorizada por emissão de fóton único (SPECT), e a ressonância magnética. As técnicas de emissão têm fornecido informações sobre a hemodinâmica e o metabolismo cerebral, além de ligação, bloqueio e disfunção de receptores. ${ }^{3}$ A literatura sobre ressonância magnética inclui um grande número de relatos envolvendo os efeitos do consumo crônico de álcool sobre a anatomia cerebral; um número menor de relatos de anormalidades cerebrovasculares relativamente infreqüentes envolvendo usuários de drogas ilícitas; e um número ainda menor de trabalhos, mas que vem crescendo rapidamente, sobre os efeitos de diversas substâncias sobre a fisiologia cerebral (com o uso de ressonância magnética funcional - RMF, pela técnica BOLD ) e neuroquímica (por meio de espectroscopia por ressonância magnética). ${ }^{4}$

\section{Cocaína}

Em relação a outras substâncias de abuso, os efeitos da cocaína sobre o fluxo sangüíneo e o metabolismo cerebral de glicose são, provavelmente, os melhor compreendidos. ${ }^{3}$ Embora seja classificada como "estimulante" (por seus efeitos comportamentais e sobre o psiquismo), a administração de cocaína foi associada com a redução global no metabolismo cerebral de glicose $^{5}$ (Figura 1) e do fluxo sangüíneo cerebral, ${ }^{6,7}$ em paralelo com os efeitos subjetivos da droga. A significativa vasoconstrição arterial cerebral induzida pela cocaína, mesmo em doses relativamente baixas, pode ser uma explicação plausível para essa aparente contradição (Figura 2). ${ }^{8} \mathrm{Um}$ estudo empregando RMF comparou os efeitos da administração de cocaína e placebo num grupo de indivíduos dependentes de cocaína. ${ }^{9}$ Foram descritas várias áreas de ativação rápida e transitória localizadas em regiões que incluíram cíngulo, córtex pré-frontal dorsolateral, prosencéfalo basal, núcleo caudado, tegmento ventral e ponte, ao passo que, foi observada uma ativação mais gradual e mantida no núcleo accumbens. Os autores relataram que as ativações iniciais e tardias correlacionaramse, respectivamente, com a euforia e com a sensação de "fissura" pela droga (craving).* Entretanto, os profundos efeitos vasculares da cocaína não podem ser desconsiderados e esses achados, ainda que importantes, devem ser considerados preliminares.

Em termos da investigação das ações da cocaína no nível molecular, há evidências provenientes de estudos de imagem de que a cocaína, em doses suficientes para desencadear euforia, liga-se de modo relativamente seletivo ao transportador de dopamina pré-sinaptico (TDA). ${ }^{10}$ Outro estudo que reforça a hipótese da importância do bloqueio do TDA para a produção dos efeitos de euforia pela cocaína estima que pelo menos metade dos sítios disponíveis de TDA devem ser ocupados para que haja efeitos comportamentais. ${ }^{11}$ Por outro lado, reconhece-se que o bloqueio do TDA não deve ser o responsável exclusivo pela euforia induzida por drogas psicoestimulantes como a cocaína, uma vez que, mesmo altas taxas de bloqueio do TDA induzidas por metilfenidato $(>80 \%)$ não impedem que doses adicionais dessa substância provoquem sensações de euforia em voluntários normais. ${ }^{12} \mathrm{O}$ envolvimento de receptores dopaminérgicos $\mathrm{D}_{2}$ pós-sinápticos na euforia induzida por cocaína é sugerido por um estudo empregando PET e $\left[{ }^{11} \mathrm{C}\right]$ racloprida (um ligante seletivo desse receptor), no qual a administração de cocaína, além de provocar euforia nos usuários da droga que participaram do estudo, também diminuiu a ligação estriatal do traçador, o que sugere que a cocaína aumentou os níveis sinápticos de dopamina, promovendo o deslocamento da racloprida marcada. ${ }^{13}$

Conseqüências do uso crônico de cocaína, geralmente feito por indivíduos com dependência, também têm sido estudadas. Os efeitos vasoativos marcantes da substância são uma explicação plausível para a incidência aumentada de acidentes vasculares cerebrais (mesmo em indivíduos relativamente jo-

\footnotetext{
*A palavra "craving" descreve a sensação de forte desejo de consumir uma substância de abuso, geralmente acompanhada por sintomas ansiosos, que cessa quando essa substância é utilizada. A expressão não tem equivalente adequado em português. Assim, foi utilizado o termo comumente empregada pelos pacientes para descrever essa sensação ("fissura"), mesmo tratando-se de gíria.
} 
vens) associados ao consumo dessa droga. ${ }^{4}$ Há evidências crescentes de que anormalidades de fluxo sangüíneo cerebral ocorrem freqüentemente em usuários de cocaína, mesmo na ausência de sintomas neurológicos evidentes ${ }^{14,15} \mathrm{e}$ já foi sugerido um efeito cumulativo decorrente de múltiplos episódios de consumo da substância. ${ }^{8}$ Estudo feito por Kosten et al não foi capaz de evidenciar anormalidades de perfusão cerebral por meio de SPECT em dependentes de cocaína, a não ser nos indivíduos que também faziam uso importante de álcool, o que sugere uma interação entre as duas substâncias. ${ }^{16}$ Entretanto, esse estudo não parece ter utilizado o melhor método para quantificar as anormalidades de fluxo sangüíneo cerebral geralmente observadas em exames de SPECT de dependentes de cocaína, que correspondem a múltiplas áreas de hipoperfusão cerebral (Figura 3). A grande maioria dos estudos encontrados na literatura descrevendo anormalidades de perfusão cerebral em dependentes de cocaína têm sua interpretação complicada pela história de uso de opióides. Entretanto, esse mesmo padrão de alterações de fluxo sangüíneo cerebral foi também descrito num estudo realizado em nosso meio, onde o consumo de drogas opióides é relativamente baixo. ${ }^{17}$ Ainda não está claro se essas anormalidades de fluxo são reversíveis ou não. Um trabalho foi capaz de evidenciar anormalidades de perfusão cerebral mesmo após períodos de até seis meses sem consumo de drogas, ${ }^{18}$ enquanto outros estudos demonstraram melhora do padrão de fluxo com tratamentos que envolveram buprenorfina (um agonista parcial opióide), ${ }^{19}$ ou drogas com atuação sobre a atividade plaquetária. ${ }^{20} \mathrm{Em}$ contraste com estudos de neuroimagem funcional, investigações envolvendo métodos estruturais têm mostrado resultados comparativamente menos exuberantes em usuários crônicos de cocaína (geralmente usuários de múltiplas substâncias, incluindo cocaína). ${ }^{4} \mathrm{Um}$ relato de que dependentes de cocaína teriam uma frequiência maior (em comparação a controles pareados por idade) de anormalidades em substância branca, detectadas por imagens pesadas em $\mathrm{T}_{2}$ de ressonância magnética, ${ }^{21}$ é comprometido pelo fato de que mais da metade da amostra estudada também apresentava diagnóstico prévio ou atual de dependência de álcool, que também está associado a essas anormalidades de substância branca.

Resultados interessantes têm sido obtidos na aplicação de técnicas de neuroimagem no estudo da "fissura" por drogas. Indivíduos dependentes de cocaína freqüentemente experimentam essa sensação quando são expostos a desencadeantes ambientais, o que é uma causa importante de recaídas. Três estudos avaliaram a fissura provocada por estimulação audiovisual (vídeo contendo imagens e sons relacionados à cocaína) em usuários ou dependentes de cocaína, comparando-os com controles normais. Um desses estudos, avaliando a ativação cerebral por meio de PET com $\left[{ }^{18} \mathrm{~F}\right]$ fluorodeoxiglicose, evidenciou aumentos de matabolismo regional de glicose no córtex pré-frontal dorsolateral e na amígdala (Figura 4), além do cerebelo. Esses aumentos de metabolismo foram correlacionados com a sensação de "fissura", desencadeada pela estimulação audiovisual nos indivíduos dependentes de cocaína, o que não ocorreu nos controles normais. ${ }^{22}$ Uma investigação semelhante empregando RMF encontrou ati- vações significantes no cíngulo anterior e no córtex pré-frontal dorsolateral esquerdo num grupo de usuários de cocaína, havendo correlação com a intensidade da "fissura" que foi relatada. ${ }^{23}$ Mais recentemente, um estudo com PET e $\left[{ }^{15} \mathrm{O}\right] \mathrm{H}_{2} \mathrm{O}$ demonstrou aumento de fluxo sangüíneo em áreas límbicas, particularmente o cíngulo anterior e a amígdala, durante a fissura e desencadeada por estimulação audiovisual em usuários de cocaína. ${ }^{24} \mathrm{Na}$ comparação entre os grupos de usuários e nãousuários de cocaína, não foram observadas diferenças significativas em termos de ativação em áreas controle (córtex pré-frontal dorsolateral, cerebelo, tálamo e córtex visual). ${ }^{24}$ As diferenças observadas nos padrões de ativação desses três estudos podem ser explicada em parte pelas diferentes técnicas de imagem empregadas. É sugerido que um sistema neuronal integrando aspectos cognitivos e emocionais da memória tenha um papel importante na ocorrência da "fissura" por cocaína. ${ }^{22}$

\section{Álcool}

Confirmando achados anatomopatológicos, a investigação com neuroimagem estrutural envolvendo dependentes de álcool, tem demonstrado alargamento de ventrículos e sulcos, perda de volume tanto de substância cinzenta quanto de substância branca cerebral, aumento do volume de líquor e redução no tamanho do corpo caloso relacionada à duração do consumo de álcool. ${ }^{25} \mathrm{~A}$ atrofia cerebelar é um dos achados mais importantes, sendo um indicador clínico importante de lesão encefálica pelo álcool. ${ }^{4}$ Também têm sido descritas alterações de substância branca (hipersinal em imagens pesadas em $\mathrm{T}_{2}$ ), mesmo em alcoolistas jovens e assintomáticos do ponto de vista neurológico. ${ }^{4} \mathrm{~A}$ atrofia cerebral, assim como a dilatação ventricular e o desempenho cognitivo parecem melhorar com a interrupção do consumo de álcool, havendo relação entre o grau de melhora e o período sem consumir a substância. ${ }^{25}$

Métodos de neuroimagem funcional para a avaliação dos efeitos do álcool têm sido empregados por décadas, mas os resultados têm sido por vezes conflitantes. De um modo geral, é aceito que o uso crônico de álcool está associado à redução do fluxo sangüíneo cerebral e do metabolismo cerebral de glicose, enquanto a ingestão aguda de etanol resulta em aumento de fluxo (possivelmente por vasodilatação induzida pelo etanol). ${ }^{3}$ Apesar do acoplamento estreito que normalmente ocorre entre fluxo sangüíneo e metabolismo cerebral, a ingestão aguda de álcool está geralmente associada à redução do metabolismo cerebral de glicose. ${ }^{26}$ Durante a abstinência, o achado mais relatado é uma redução global do metabolismo de glicose e do fluxo sangüíneo cerebral, sugerindo que ambos os parâmetros ficam acoplados nessa situação clínica. ${ }^{25}$

\section{Outras drogas}

Dados referentes à investigação por neuroimagem das ações de outras drogas de abuso são relativamente mais escassos, com exceção dos opióides, cujo uso é relativamente infreqüente em nosso meio.

\section{Opióides}

A intoxicação aguda está associada a reduções globais de 
metabolismo de glicose e fluxo sangüíneo cerebral; a abstinência foi associada a múltiplas falhas de perfusão cerebral em avaliações feitas por SPECT na primeira semana após a interrupção do uso de drogas, com certa recuperação nos exames realizados duas semanas depois. ${ }^{26}$

\section{Maconha}

É uma das drogas mais comumente abusadas, embora relativamente pouco estudada até o momento. Os efeitos no fluxo sanguiíneo cerebral do tetra-hidrocanabinol (THC), a principal substância psicoativa presente na planta, parecem depender da experiência do indivíduo: fumantes experientes apresentam aumento de fluxo (geralmente associado com sensação de relaxamento), enquanto fumantes inexperientes tendem a manifestar redução de fluxo (associada a relatos de ansiedade e depressão). Embora a resposta global no metabolismo cerebral de glicose seja variável, após a administração intravenosa de THC, é relatado aumento da atividade do cerebelo, região que concentra a maior quantidade de receptores canabinóides CB1; usuários crônicos parecem apresentar fluxo sangüíneo e metabolismo cerebral de glicose reduzidos, em comparação com controles normais. ${ }^{3}$

\section{Tabaco}

Estudo com PET demonstrou redução da atividade cerebral da monoamina-oxidase A e B. É conjecturado se um aumento resultante na dopamina de sinapses não seria o responsável pelo potencial de dependência do tabaco, em vez da ação da nicotina sobre receptores colinérgicos. ${ }^{26}$

\section{Solventes e tolueno}

Estudos funcionais sobre os efeitos dessas substâncias são prejudicados pelo fato desses indivíduos muitas vezes apresentarem anormalidades estruturais. Relatos de casos descrevendo padrão de anormalidades multifocais de fluxo sangüíneo cerebral são obscurecidos pelo uso combinado de outras substâncias. Existem estudos descrevendo conseqüências da exposição ocupacional ao tolueno, evidenciando reduções discretas no fluxo sangüíneo cerebral, porém proporcionais à quantidade de exposição à substância. ${ }^{3}$

\section{Discussão}

As diferentes modalidades de exames de neuroimagem têm fornecido informações muito relevantes para o avanço do co- nhecimento das interações entre as diversas substâncias de abuso e o SNC. Há um grande potencial de que esse conhecimento acumulado venha permitir o desenvolvimento de novas abordagens em nível de tratamento de dependência e até mesmo de prevenção ao uso indevido de substâncias psicoativas. Além de confirmar em humanos alguns achados de pesquisas realizadas com outros animais, os métodos de neuroimagem abrem a perspectiva de estudo direto de ações de diversas substâncias no SNC, particularmente correlacionandoas com efeitos subjetivos, por meio de técnicas relativamente pouco invasivas.

Algumas limitações potenciais desse tipo de investigação devem ser lembradas, entretanto. Outros fatores, além do uso de substâncias psicoativas, podem estar relacionados às diferenças observadas entre usuários de drogas e controles normais. De modo geral, tem sido demonstrado que, em comparação com não-usuários, indivíduos que fazem uso de substâncias psicoativas apresentam maiores taxas de comorbidade psiquiátrica, doenças clínicas (incluindo, dentre outras, cirrose, hepatite, doenças cardiovasculares, diabetes, infecção pelo HIV/Aids e desnutrição), uso lícito ou ilícito de medicamentos e escores de quociente intelectual (QI) abaixo da média. ${ }^{27}$ Muitos relatos não definem bem a população estudada, que pode incluir indivíduos com uso esporádico, abuso (uso nocivo) ou dependência de substâncias. Muitos indivíduos que consomem álcool e outras drogas fazem uso combinado de diversas substâncias, o que torna mais complicado atribuir as alterações observadas a uma determinada substância em particular. Determinadas áreas cerebrais são estimuladas por certos estados cognitivos e emocionais, assim como por atividade motora, o que pode confundir alguns resultados. Por exemplo, um usuário de drogas pode ficar frustrado ao não experimentar efeitos desejados de uma substância ao receber placebo, assim como um voluntário normal pode apresentar ansiedade e mesmo euforia, antecipando o que seria uma nova experiência. ${ }^{26}$ É comum estudos de neuroimagem avaliarem amostras relativamente pequenas, o que os torna mais vulnerávais a erros tipo I ("falsos positivos”, em função da eventual existência de valores extremos - "outliers" - ou de vieses de amostragem) e erros tipo II ("falsos negativos", decorrentes de falta de poder estatístico). Felizmente, as pesquisas na área têm sido aprimoradas no sentido de um melhor controle sobre várias dessas dificuldades e um corpo de conhecimento progressivamente mais consistente vem se formando.

\section{Referências}

1. World Health Organization. Trends in substance use and associated health problems. Fact Sheet N 127. Agosto de 1996. Disponível em http://www.who.int/inf-fs/en/fact127.html >. Acesso em 18 de dezembro de 1998.

2. Almeida Filho N, Mari JJ, Coutinho E, França JF, Fernandes J, Andreoli $\mathrm{SB}$, et al. Brazilian multicentric study of psychiatric morbidity. Methodological features and prevalence estimates. B J Psychiatry 1997;171:524-9.
3. Levin JM. Emission tomographic studies in substance abuse. In Kaufman MJ, editor. Brain imaging in substance abuse: research, clinical, and forensic applications. Totowa (NJ): Humana Press; 2001. p. 113-54.

4. Kaufman MJ, Levin JM. Magnetic resonance findings in substance abuse. In: Kaufman MJ, editor. Brain imaging in substance abuse: research, clinical, and forensic applications. Totowa (NJ): Humana Press; 2001. p. 155-98. 
5. London ED, Cascella NG, Wong DF, Phillips RL, Dannals RF, Links JM, et al. Cocaine-induced reduction of glucose utilization in human brain. Arch Gen Psychiatry 1990;47:67-74.

6. Pearlson GD, Jeffrey PJ, Harris GJ, Ross CA, Fischman MW, Camargo EE. Correlation of acute cocaine-induced changes in local cerebral blood flow with subjective effects. Am J Psychiatry 1993;150:495-7.

7. Wallace EA, Wisniewski G, Zubal G, Vandyck CH, Pfau SE, Smith EO, et al. Acute cocaine effects on absolute cerebral blood flow. Psychopharmacol 1996;128:17-20.

8. Kaufman MJ, Levin JM, Ross MH, Lange N, Rose SL, Kukes TJ, et al. Cocaine induced cerebral vasoconstriction detected in humans with magnetic resonance angiography. JAMA 1998;279:376-80.

9. Breiter HC, Gollub RL, Weisskoff RM, Kennedy DN, Makris N, Berke JD, et al. Acute effects of cocaine on human brain activity and emotion. Neuron 1997;19:591-11.

10. Malison RT, Best SE, Wallace EA, Mccance E, Laruelle M, Zoghbi $\mathrm{SS}$, et al. Euphorigenic doses of cocaine reduce [ $\left.{ }^{123} \mathrm{I}\right] \beta-\mathrm{CIT}$ SPECT measures of dopamine transporter availability in human cocaine addicts. Psychopharmacol 1995;122:358-62.

11. Volkow ND, Wang GJ, Fischman MW, Foltin RW, Fowler JS, Abumrad NN, et al. Relationship between subjective effects of cocaine and dopamine transporter occupancy. Nature 1997;386:829-30.

12. Volkow ND, Wang GJ, Fowler JS, Gatley SJ, Ding YS, Logan J, et al. Relationship between psychostimulant-induced "high" and dopamine transporter occupancy. Proc Natl Acad Sci USA 1996;93:10388-92.

13. Schlaepfer TE, Pearlson GD, Wong DF, Marenco S, Dannals RF. PET study of competition between intravenous cocaine and $\left[{ }^{11} \mathrm{C}\right]$ raclopride at dopamine receptors in human subjects. Am J Psychiatry 1997;154:1209-13.

14. Volkow ND, Mullani N, Gould KL, Adler S, Krajewski K. Cerebral blood flow in chronic cocaine users: a study with positron emission tomography. Br J Psychiatry 1988;152:641-8.

15. Holman BL, Carvalho PA, Mendelson J, Teoh SK, Nardim R, Hallgring $\mathrm{E}$, et al. Brain perfusion is abnormal in cocaine-dependent polydrug users: a study using technetium-99m-HMPAO and ASPECT. J Nucl Med 1991;32:206-10
16. Kosten TR, Cheeves C, Palumbo J, Seibyl JP, Price LH, Woods SW. Regional cerebral blood flow during acute and chronic abstinence from combined cocaine-alcohol abuse. Drug Alcohol Depend 1998;50:187-95.

17. Nicastri S, Buchpiguel CA, Andrade AG. Anormalidades de fluxo sangüíneo cerebral em indivíduos dependentes de cocaína. Rev Bras Psiquiatr 2000;22:42-50.

18. Strickland TL, Mena I, Villanueva-Meyer J, Miller BL, Cummings J, Mehringer CM, et al. Cerebral perfusion and neuropsychological consequences of chronic cocaine use. J Neuropsychiatry Clin Neurosci 1993;5:410-27.

19. Levin JM, Mendelson JH, Holman BL, Teoh SK, Garada B, Schwartz RBE, et al. Improved regional cerebral blood flow in chronic cocaine polydrug users treated with buprenorphine. J Nucl Med 1995;36:1211-5.

20. Kosten TR. Pharamcotherapy of cerebral ischemia in cocaine dependence. Drug Alcohol Depend 1998;49:133-44.

21. Bartzokis G, Goldstein IB, Hance DB, Beckson M, Shapiro D, Lu PH, et al. The incidence of $\mathrm{T}$ - weighted MR imaging signal abnormalities in the brain of cocaine-dependent patients is age-related an regionspecific. Am J Neuroradiol 1999;20:1628-35.

22. Grant S, London ED, Newlin DB, Villemagne VL, Liu X, Contoreggi C, et al. Activation of memory circuits during cue-elicited cocaine craving. Proc Natl Acad Sci USA 1996;93: 12040-5.

23. Maas LC, Lukas SE, Kaufman MJ, Weiss RD, Daniels SL, Rogers VW, et al. Functional magnetic resonance imaging of human brain activation during cue-induced cocaine craving. Am J Psychiatry 1998;155:124-6.

24. Childress AR, Mozley PD, McElgin W, Fitzgerald J, Reivich M, O'Brien CP. Limbic activation during cue-induced cocaine craving. Am J Psychiatry 1999;156:11-8.

25. Netrakom P, Krasuki JS, Miller NS, O'Tuama LA. Structural and functional neuroimaging findings is substance-related disorders. Psychiatr Clin North Am 1999;22:313-29.

26. Gattley SJ, Volkov N. Addiction and imaging of the living brain. Drug Alcohol Depend 1998; 51:97-108.

27. Bauer LO. Electroencephalographic studies of substance use and abuse In: Kaufman MJ, editor. Brain imaging in substance abuse: research, clinical, and forensic applications. Totowa (NJ): Humana Press; 2001 p. 77-112.

\section{Correspondência: Sergio Nicastri \\ GREA}

R. Dr. Ovídio Pires de Campos, s/n - 05403-010 São Paulo, SP - Tel.: (0xx11) 3081-8060 Fax: (0xx11) 3064-4973

E-mail: snicastr@hcnet.usp.br 
Neuroimagem e substâncias psicoativas - p. 28-31

Figura 1 - Imagens de PET evidenciando redução global no metabolismo cerebral de glicose induzida pela administração de $40 \mathrm{mg}$ de cocaína intravenosa, em comparação com a administração de solução salina (placebo). 0 mesmo indivíduo realizou cada avaliação em dois momentos diferentes.

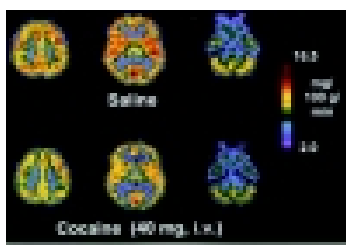

Figura 2 - Angiografia por ressonância magnética evidenciando vasoconstrição em artérias cerebrais. Ambas as imagens são provenientes de um mesmo indivíduo, realizadas antes (à esquerda) e 20 minutos após (à direita) a administração de uma dose de $0,4 \mathrm{mg} /$ kg de cocaína intravenosa. As setas superiores indicam as artérias cerebrais médias $e$ as setas inferiores apontam para as artérioas cerebrais posteriores. $A=$ anterior; $P=$ posterior; $\mathbf{R}=$ direito; $L=$ esquerdo.
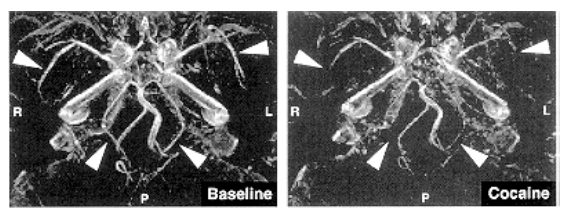

Figura 3 - Imagens de SPECT evidenciando múltiplas áreas de hipofluxo sangüíneo cerebral em dependente de cocaína do sexo masculino, de 20 anos de idade, abstinente de drogas há três semanas.

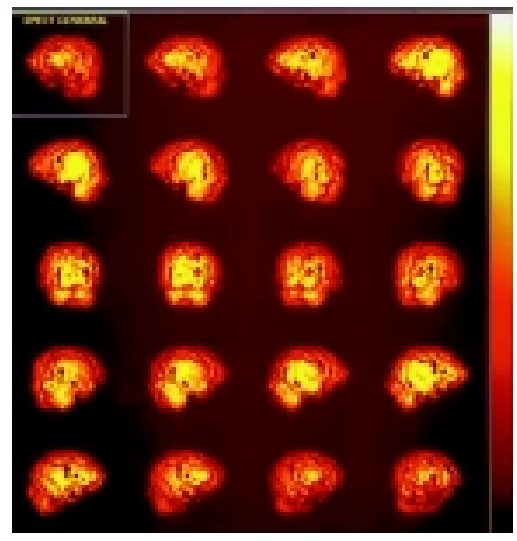

Figura 4 - Imagens de corregistro de PET e ressonância magnética evidenciando áreas de aumento do metabolismo cerebral de glicose em indivíduo com abuso de cocaína em resposta a estimulação audiovisual relacionada a cocaína. As setas indicam o córtex pré-frontal dorsolateral (DL) e a amígdala (AM), ativados durante a "fissura".

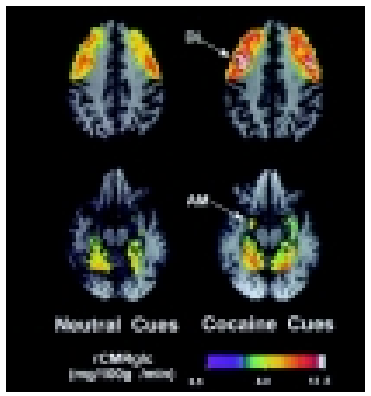

